\title{
Une revue périodique en endoscopie digestive doit-elle suivre ou accompagner les mutations de la profession?
}

\section{Heresbach}

(C) Springer-Verlag 2009

Il y a quelque temps, les revues ou périodiques médicaux, en particulier consacrés à l'endoscopie digestive, ne comportaient que des articles originaux, des cas cliniques ou des images rares destinés à la diffusion de la connaissance et à la formation continue des endoscopistes. Depuis quelques années, notamment dans les revues de langue anglaise, sont apparus des articles ayant plus trait à l'épidémiologie des maladies, mais également aux modalités et conditions de réalisation de l'endoscopie ou aux recommandations concernant l'exercice de l'endoscopie. La notion de recommandation ou de consensus professionnel, en particulier dans les revues anglo-saxonnes et américaines, se juxtapose rapidement à l'aspect médicolégal qui dans ces pays ne s'inquiète pas de la subtilité française pour savoir si le consensus ou les recommandations sont opposables ou non. Il est clair que cette orientation est dictée par les modalités d'exercice, en particulier de l'endoscopie digestive et des conséquences financières ou légales en cas de complications ou d'effets secondaires qui, encore une fois en France, sont subtilement divisés en effets indésirables graves ou non graves et surtout attendus ou inattendus !

Faut-il suivre cette évolution dans les revues de langue française, en particulier dans Acta Endoscopica, revue officielle de la SFED, en publiant, à côté des articles originaux ou de formation médicale continue dont la portée est scientifique et technique, des articles originaux ou des avis d'experts dont la portée sera plutôt économique et juridique ?

À l'heure de la diminution du produit national brut, et donc du produit national brut per capita, les quérulences risquent d'être plus fréquentes et surtout d'avoir pour objectif non pas un dédommagement moral mais bel et bien un dédommagement financier, comme en ont témoigné récemment l'évolution des primes de Responsabilité civile

D. Heresbach $(\bowtie)$

Rédacteur en chef

Service des maladies de l'appareil digestif

CHU de Pontchaillou, F-35033 Rennes cedex 9

E-mail : denis.heresbach@ chu-rennes.fr professionnelle et «la période des soldes » sur ces primes à l'ère de l'évaluation des pratiques à risque...

Vous comprendrez que la place faite dans ce numéro, d'une part au taux de redevance des gastroentérologues, à leur institution ou clinique, analysée à la lumière des prestations fournies par ces dernières, ainsi que les avis d'experts visant à décoder le langage et les procédures juridiques, témoigne que cet aspect de l'endoscopie doit être développé non seulement dans un but de formation médicale continue mais également et surtout pour pérenniser la qualité et la diversité de l'activité endoscopique. Cet aspect de la revue n'empêche pas de publier dans ce même numéro les textes de «l'état de l'art» et des «ateliers de Video Digest $2009 »$ qui pour la première fois seront disponibles lorsque vous assisterez à Video Digest.

Nous disposons en France de trop d'avis d'experts ou de jugements ex abrupto, à l'inverse d'autres pays, et nous ne pouvons qu'encourager la publication dans Acta Endoscopica d'articles originaux de type enquête transversale et, encore mieux, longitudinale et prospective, sur les modalités techniques et économiques de l'exercice de l'endoscopie, qui fourniront les éléments nécessaires à faire valoir auprès de nos institutions publiques ou privées, voire politiques, ou les prérequis de l'exercice de l'endoscopie pour respecter l'obligation de moyen qui, in fine, est la seule qui demeure légalement opposable.

En espérant qu'en tant que lecteur vous ne prendrez pas cet éditorial pour un aléa ou une «maladresse », nous souhaitons que l'arrêté de la Première chambre civile de la Cours de cassation du 10 septembre 2008 stipulant que « la coloscopie était un acte à visée exploratoire dont la réalisation n'impliquait pas une atteinte aux parois des organes examinés, la perforation étant la conséquence d'un "geste maladroit" ", en excluant la notion d'aléa thérapeutique, ne sera qu'une péripétie et que l'ensemble des acteurs de la profession (société savante et syndicat) trouvera le moyen d'établir un dossier destiné à stigmatiser le caractère arbitraire et infondé de ce jugement, qui pour le coup est à la fois aléatoire et maladroit. 